International Journal of Engineering \& Technology, $7(3.27)(2018)$ 179-181
International Journal of Engineering \& Technology
WPC
Website: www.sciencepubco.com/index.php/IJET
Research paper

\title{
Survey on Image Dimensionality Reduction Using Deep Learning Techniques
}

\author{
K.M. Monica ${ }^{*}$, G. Bindu ${ }^{2}$, S. Sridevi ${ }^{3}$ \\ ${ }^{I}$ Department of Computer Science \& Engineering, Vels Institute of Science, Technology \& Advanced Studies(VISTAS), Pallavaram, \\ Chennai. \\ ${ }^{2}$ Department of Computer Science \& Engineering, Vels Institute of Science, Technology \& Advanced Studies(VISTAS), Pallavaram \\ Chennai. \\ ${ }^{3}$ Department of Computer Science \& Engineering, Vels Institute of Science, Technology \& Advanced Studies(VISTAS), Pallavaram, \\ Chennai. \\ *Corresponding author E-mail:monica.se@velsuniv.ac.in
}

\begin{abstract}
Images provide rich information. With reference to the data set which may be related or unrelated in nature, locates step by step, a wide range of application and its attributes through capturing mechanism by sensing the suitable technologies. On the other hand, it also creates a huge quantity of data which may be relevant, irrelevant or redundant in nature and it is used for detailed task of the image. Also, Many brings a lot of problems such as increase in computational time of image, density of image and range of mapping of data, semantics of the data set and also it also there is a scope of huge amount of labeled data for the process of training to the new environment setup. Mostly, this is not easy and costly for users to obtain sufficient training models in several application modules. This research paper deals with these problems by exploring the more classical dimension reduction algorithms with deep knowledge for supporting communities.
\end{abstract}

Keywords: Image, dimensional reduction, deep learning, real time application

\section{Introduction}

In several specific areas, like economics and artificial intelligence, the data demonstration study is an important way to make possible successive classification, recovery and suggestion task. Normally, it is vital and challenging to discover the important information from data and to discover about the primary data which is very much needed. On account of this, machine learning group has initiated the research for diverse learning, which would ascertain the essential structure of data with high dimension. From the observation of the data formulation, an algorithm may be considered as linear or nonlinear or it may be global or local.

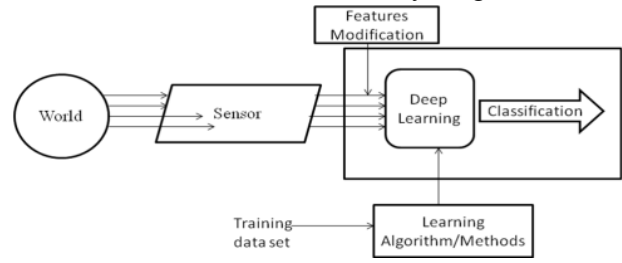

Fig. 1: Overview of deep learning activities

In the past few decades, Principle Component Analysis (PCA) and Linear Discriminate Analysis (LDA) are the traditional dimensional reduction methods that are studied extensively. Deep learning a part of ML and a special type of artificial neural network (ANN), which is similar to multilayered human cognition system. Nowadays, Deep learning is in advance for its utilization in health care data.

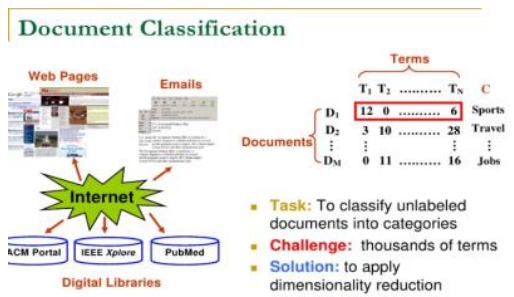

Fig. 2: Document classification

Applications of dimensionality reduction

1. Customer relationship management

2. Text Mining

3. Image retrieval

4. Protein classification

5. Image Recognition

6. Handwritten digital recognition

7. Intrusion Reduction

\section{Deep Learning Models}

Since the available data is few and parameters volume is high, the multilayer and the Neural Networks training data are easy to combine in a local optimum. With a proper structural design, the computed gradient that is near to the input layer will be normally very minute to train the deep models using the Back Propagation algorithm. The following metrics as give below

- No need to figure out the features ahead of time. 
- Same neural net approach is used for different problem.

- Fault Tolerant

\section{Support Vector Mechanism}

A deep three layered architecture is used for deep learning of data. The features of deep learning made advantages because of few aspects like large labeled data availability, feature learning and General purpose graphics processing units (GPGPU). Algorithms are listed below which is widely applied in all real time problems.

- Linear classification

- Regression

- Decision Tree

- Bayesian

- Clustering

- Association Rules.

\section{Literature Review}

Literature survey is a review with a collection of theoretical and methodological contributions among the selected area in the research, where the researcher has limited experience and decisive examination. Comparison between various algorithms is done to have better understanding. To update with the past data and data sources, literature review can be used to include the current knowledge including substantive.

Guoqiang Zhong et al (2016), here the analysis on data representation learning, feature learning and recent deep learning is surveyed. This analysis summarized that deep learning is not a new concept but it is an actual research outcome of methods like feature learning methods and Artificial Neural Networks. The detailed study of deep learning not only affects the artificial intelligence area, but it greatly improves the progress of many domains, such as finance and bioinformatics. At the end of this research it is highlighted that deep learning is not the whole thing of machine learning but it is just the way to understand artificial intelligence. [1]

Haozhe Xiea et al (2017), Random Projection is a powerful dimensionality reduction technique that has been developed and matured during the past 15 years. With the rapid increase of big data, RP has provided tangible benefits to counteract the burdensome computational requirements and has met the needs of real-time processing in some situations. Despite the fact that RP is computationally efficient, it often introduces relatively high alteration. To solve this problem, major successful have been made to improve the performance of RP, as summarized in this survey. The features cannot be well-represented in the high dimensional space produced by extant methods, such as rectangle filters and ELM. Therefore, the proposal of accurate dimensionality increasing approaches constitutes a promising direction for future RP research.[2]

Surajit Saikia et al (2017), this paper detects object related to indoor environment (i.e. bedroom) as a real time system and the algorithm used in this paper is R-CNN algorithm, which can detect the objects among the network itself. This algorithm is widely used to develop real time object detection applications because of its property used in the paper. We have created a testset "Image Net-Room Objects" for evaluating this system comprising of images commonly found in an indoor environment, and accuracy is achieved at the end. [3][8]

Sachee Nene (2017), Deep learning is the most emerging field of machine learning and has shown extensive contribution in various research areas. It has helped in overcoming the drawbacks of traditional methods by making the systems less complex and fast. Since deep has a solid practical background, strong theory based fundamentals also need to be established. Deep learning has been used with NLP in several research areas, which is quite promising and is a success. Though the outcomes are still in the rudimentary stages with some research areas, the systems understand the human language more better. This requires a more massive research on both deep learning and NLP. [4]

June-Goo Le et al (2017), Use of deep learning and AI in radiology is currently in the stages of infancy. One of the most important factors for the development of $\mathrm{AI}$ and its proper clinical adoption in radiology would be a good mutual understanding of the technology, and the most appropriate form of radiology practice and workflow by both radiologists and computer scientists/engineers. In recent technological innovations by Image Net, large and fully annotated databases were used for advancing AI development in medical imaging. This is important for training the deep learning network, and also for its evaluation. The active involvement of many radiologists is also essential for establishing a large medical imaging database. Furthermore, there are various other issues and technical problems to solve and overcome. Finally, ethical, regulatory, and legal issues raised in the use of patient clinical image data for the development of AI should be carefully considered. [5]

Murinto and Nur Rochmah Dyah PA(2017), In this research paper a hybrid method is used for reduction in dimensionality of hyper data. This also uses Support Vector Machine (SVM) and Discriminate Independent Component Analysis (DICA). In this experiment $\mathrm{KNN}$ is applied for the classification. Classification is done by using SVM and DICA on AVIRIS dataset, the value of AA is 0.7527 , OA is 0.7901 , and $K$ is 0.7608 . Hence this is considered to be the appropriate method for reducing the data dimension on an image. Higher accuracy is obtained with the classification process when compared to previous method such as PCA, ICA and DICA. Therefore this method can be used for image segmentation based on bio-inspired algorithm for hyper spectral image. [6]

\section{Conclusion}

Feature selection (e.g., mutual information, minimal redundancy maximal relevance) and Feature extraction are used to remove the capacity of dataset, so that relevant data are used to make a base for the classification method Support Vector Machine (SVM). From literature survey it is concluded that PCA yields the most capable performance in reducing the number of features or spectral bands. It is noted that in order to reduce the complexity in the computation, the method that is proposed can achieve better classification results over the SVM on a small volume of training dataset which makes it suitable for working with real-time applications or it can also be used with a limited training data. It can also achieve better performance related to SVM algorithm on large datasets where it takes less computing time.

\section{References}

[1] Zhong G, Wang LN, Ling X \& Dong J, “An overview on data representation learning: From traditional feature learning to recent deep learning", The Journal of Finance and Data Science, Vol.2, No.4,(2016), pp.265-278.

[2] Xie H, Li J \& Xue H, "A survey of dimensionality reduction techniques based on random projection", arXiv preprint arXiv: 1706.04371, (2017).

[3] Saikia S, Fidalgo E, Alegre E \& Fernández-Robles L, “Object detection for crime scene evidence analysis using deep learning", International Conference on Image Analysis and Processing, (2017), pp.14-24

[4] Surendar, A. (n.d.). Short communication: Role of Microbiology in the Pharmaceutical \&Medical Device. 433| International Journal of Pharmaceutical Research, 10(3).

[5] Lee JG, Jun S, Cho YW, Lee H, Kim GB, Seo JB \& Kim N, "Deep learning in medical imaging: general overview", Korean journal of radiology, Vol.18, No.4,(2017), pp.570-584.

[6] Murinto \& Dyah NR, "Dimensionality Reduction using Hybrid Support Vector Machine and Discriminant Independent Component Analysis for Hyperspectral Image", International Journal of 
Advanced Computer Science and Applications, Vol.8, No.11,(2017), pp.601-605.

[7] Su J, Yi D, Liu C, Guo L \& Chen WH, "Dimension reduction aided hyperspectral image classification with a small-sized training dataset: experimental comparisons", Sensors, Vol.17, No.12, (2017).

[8] B Kassimbekova, G Tulekova, V Korvyakov (2018). Problems of development of aesthetic culture at teenagers by means of the Kazakh decorative and applied arts. Opción, Año 33. 170-186 